\title{
Study on a Motor Driver for an Improved SRM by Using Constant Current Power Supply
}

\author{
Hitoshi Miyata and Eikou Gonda \\ Department of Electrical and Computer Engineering, National Institute of Technology, Yonago College \\ 4448, Hikona-cho, Yonago-shi, Tottori-ken, 683-8502 Japan
}

Hitoshi Miyata: miyata@yonago-k.ac.jp

\begin{abstract}
This paper describes a novel motor which is an improved SRM (Switched Reluctance Motor) and a study of its driving circuit. Our objective is applying the motor to the $\mathrm{EV}$ (Electric Vehicles) for one of the solution of the rare earth issue. In order to realize it, control method of the motor and driving circuits must be developed. For the control method, DC chopper, phase controller and inverter are proposed so far. On the other hand, SRM has strong nonlinearity and some disadvantages. Due to the nonlinearity, computer simulation of the motor and its driving circuits are quite difficult. Therefore, we had to evaluate our method by experiments using real hardware. In this paper, at first, structural feature of the novel motor is explained. Then a principle of commutation circuit will be described. In addition, this paper presents a structure and operation of constant-current power supply which plays important role for control of the novel motor. Finally, results of experiments of the motor drive by using our driving circuits will be presented.
\end{abstract}

Keywords: Improved SRM, Rare Earth, EV.

\section{Introduction}

Hybrid and Electric Vehicle are receiving a lot of attention instead of Internal Combustion Engine Vehicle recently ${ }^{(1-4)}$. In Japan, many automobile manufacturers have begun to make and sell Electric Vehicle (EV). Almost all motors applied to the EV, in Japan, are Interior Permanent Synchronous Motor (IPMSM) ${ }^{(5-7)}$. Neodymium which is one of the so called rare earth is employed as a magnet material of the IPMSM to realize high efficiency and high output. As rare earth is unevenly distributed in small amount on our planet, reliable supply is concerned in the near future. In addition, it is very expensive.

Based on such a backdrop, we believe that applying the novel motor to the $\mathrm{EV}$ enables us to produce the $\mathrm{EV}$ at lower cost. The driving method of the novel motor is similar to that of Switched Reluctance Motor (SRM) ${ }^{(8,9)}$. Disadvantages of the SRM are torque ripple, laud noise during the operation and so on. The new motor can be reduced those disadvantages by employing multilayer architecture. On the other hand, as the motor has strong nonlinear characteristics, we have to make experiments by using real circuit without computer simulation.

\section{Motor Without Rare Earth Materials}

\subsection{Outline and Structure of the Motor}

The novel motor is a developed type of SRM. The motor can reduce the noise, vibration and torque ripple. Fig. 1 indicates a cross section of the motor. The rotor and the stator are made of electrical steel and both are salient pole structure. Only the stator has coil winding which is concentrated winding. The motor comprises 8 poles stator and 4 poles rotor. Fig. 1 illustrates the motor has 2 phase windings which include phase $\mathrm{A}$ winding and phase $\mathrm{B}$ winding. Fig. 2 shows the motor has a multilayered structure. In this case, stator and rotor consist of multilayer components along the rotation axis direction.

\subsection{Reduction of Noise and Vibration}

In the case of conventional SRM, noise and vibration are occurred during operation. The cause of 


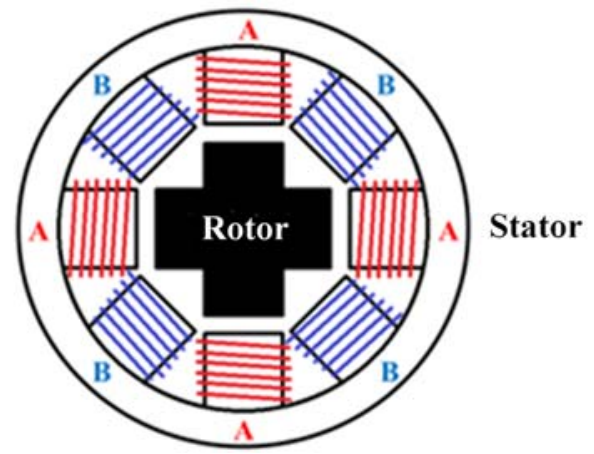

Fig. 1. A cross section of a novel motor.

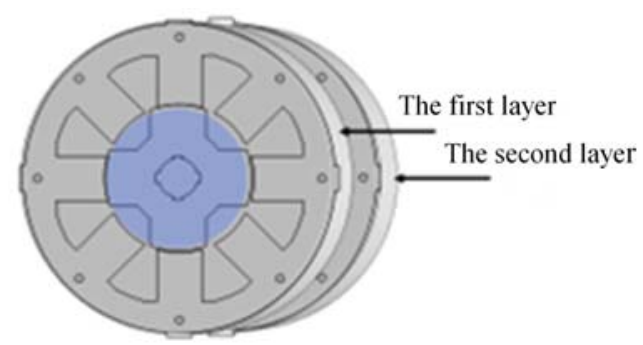

Fig. 2. A multilayered structure.

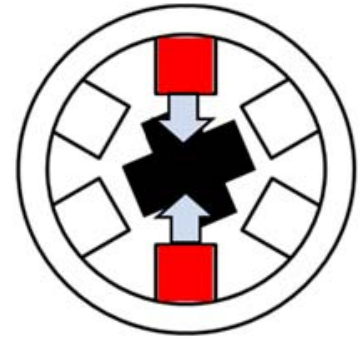

(a) SRM

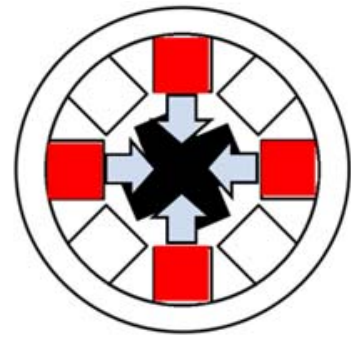

(b) The novel motor
Fig. 3. Force of compressive deformation of the stator.

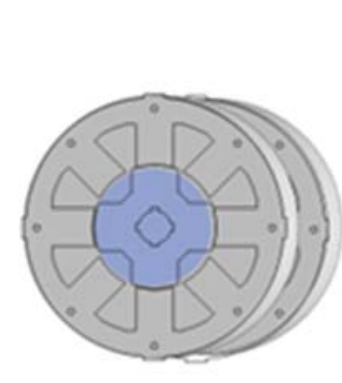

Rotor angle of inclination

(b) Front view

(b) Rotor angle of inclination

Fig. 4. A cross section of the motor.

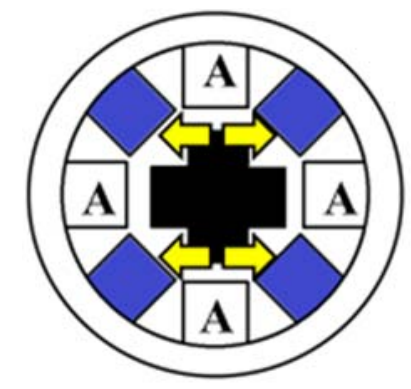

Fig. 5. In the case of the motor can't rotate.

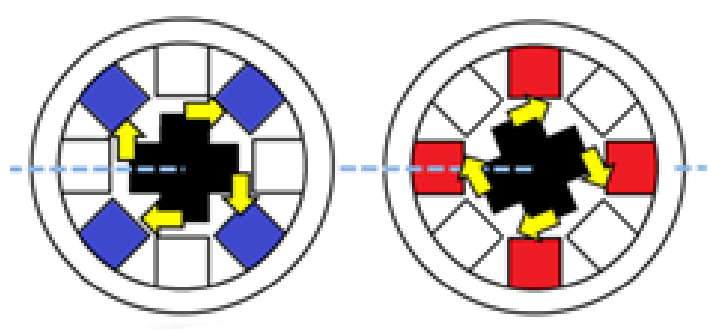

$\begin{array}{ll}\text { (a) The first layer } & \text { (b) The second layer }\end{array}$

Fig. 6. Determination of the rotation direction.

those phenomena is compressive deformation. Fig. 3(a) illustrates compressive forces of SRM. The stator of the new motor is also deformed by attraction toward the rotor when the stator is excited. But, as Fig. 3(b) shows, in the case of the novel motor, the forces of compressive deformation are mutually canceled. As a result, deformation of the stator which is the cause of the noise and vibration is considerably reduced.

\subsection{Multilayer Architecture}

The greatest feature of the novel motor is a multilayer architecture as Fig. 4 shows. The conventional motor consists of only one layer stator and rotor. In the case of the novel motor, two layers or more are laminated along the rotation axis direction. The second layer and after that are rotating according to rotor angle of inclination. The rotor angle of inclination is different depending on the number of layers. This architecture enables to determine the rotation direction of the motor and to eliminate torque ripple. Determination of the rotating direction is carried out as follows;

Fig. 5 indicates only one layer of the stopping motor whose phase A winding and salient pole of the rotor are in the confronting position. In this case, as current flows in 


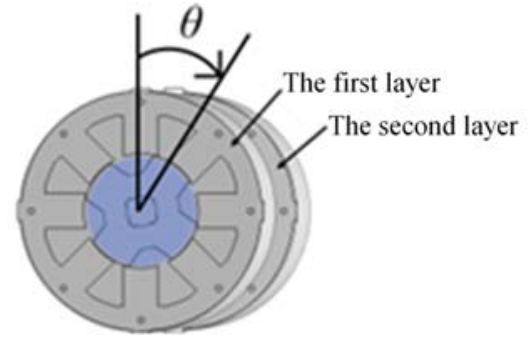

(a) Definition of $\theta$

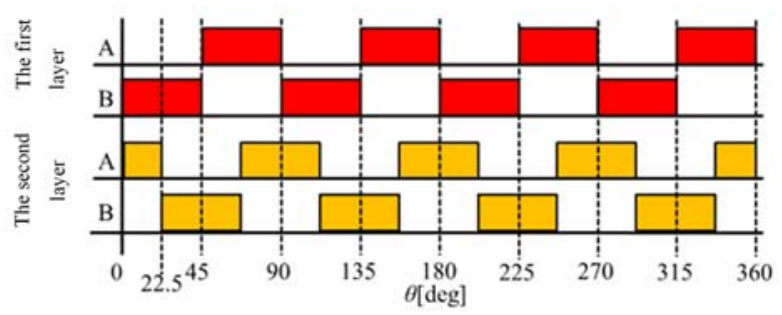

(b) Timing of the current

Fig. 7. In the case of the motor can't rotate.

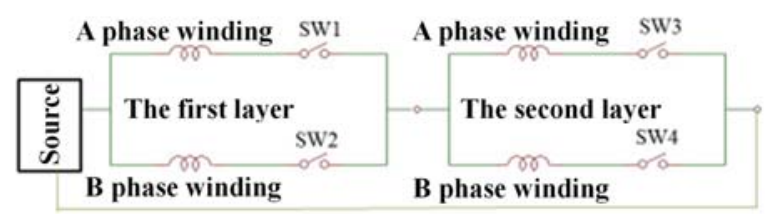

Fig. 8. Determination of the rotation direction.

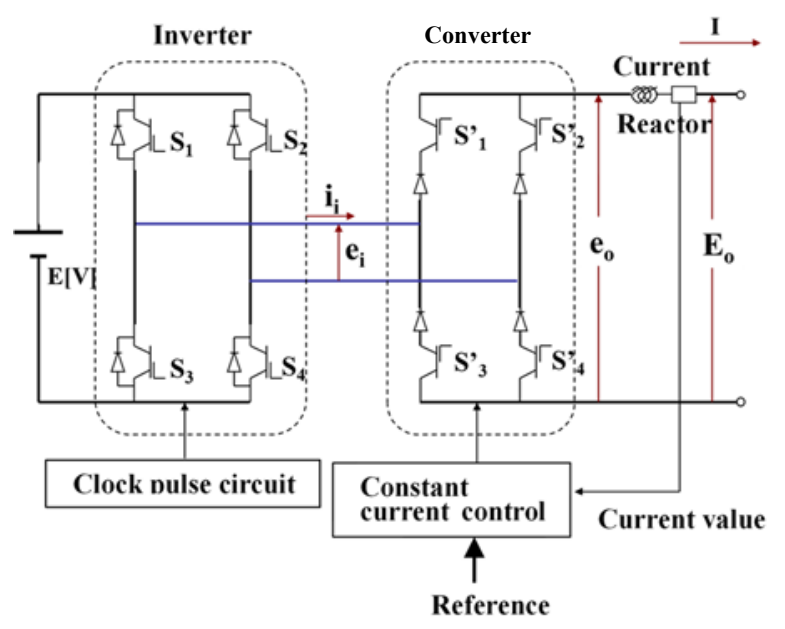

Fig. 9. Phase controlled constant current power supply. the winding of the painted pole, in Fig. 5, the rotor is applied clockwise torque and the stator is applied counterclockwise torque. As those torque cancels each other, the rotor never rotate. Fig. 6 illustrates multiple layer stator and position of the rotor and added second layer. At this time, position of the rotor of the second layer is in the middle of phase A winding and phase B winding. As a result, in order to rotate the motor in the clockwise direction, the phase A winding painted in Fig. 6 is excited. Fig. 7 illustrates in the case of the motor rotates in the clockwise. Fig. 7(a) indicates the definition of inclination angle $\theta$. Fig. 7(b) designates the timing of current flows.

\section{Driving Circuits for the Novel Motor}

\subsection{Commutation Circuit}

As the rotor angles of the novel motor are different from each other, current flows each phase is switched separately. A commutation circuit is employed for the operation. The commutation circuit consists of the windings and semiconductor switches. Fig. 8 shows A and $\mathrm{B}$ windings connected in parallel for each layer. The current of each winding is controlled by each switch. On the other hand, those layers are connected in serial in order to equalize current and torque generated at each layer. The torque of the motor is controlled by the current which flows each winding.

\subsection{Constant Current Power Supply}

In order to drive the novel motor, constant current power supply which keeps supplying current arbitrarily specified value is used. Employing constant current power supply makes sense, because the torque of the motor is controlled by current flow in the winding.

The current of the power supply is in one direction. The circuits of the power supply can be based on DC chopper or phase control method. Fig. 9 illustrates a circuit of phase control method, where $i_{i}$ and $e_{i}$, are current and voltage between inverter and converter, $e_{0}$ is the output of the converter and $E_{\mathrm{o}}$ is the voltage of the constant current power supply. The power supply consists of inverter, converter, DC power supply, clock pulse circuit, constant current control circuit, reactor and capacitor. The inverter and the converter comprise four semiconductor switches each. The switches for the inverter $S_{1}, S_{4}$ and $S_{2}, S_{3}$ are operated synchronously by the control signal of clock pulse circuit. The $S{ }_{1}, S^{\prime}{ }_{4}$ and 


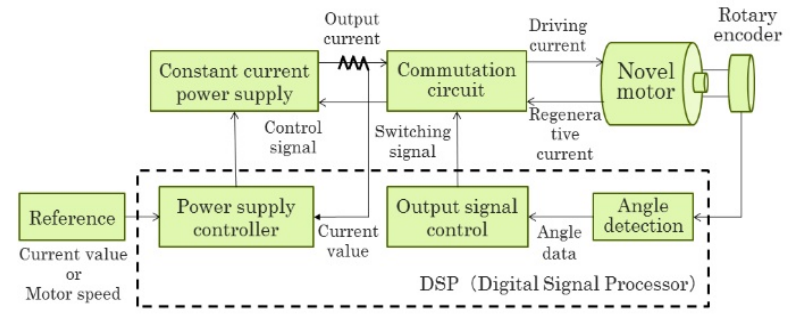

Fig. 10. Driving circuit of the novel motor.

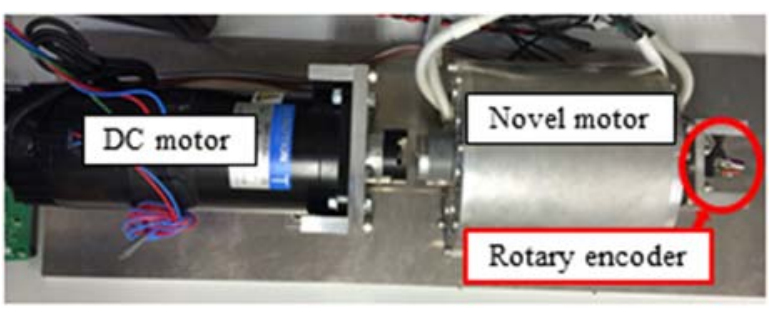

Fig. 11. Experimental equipment of the novel motor.

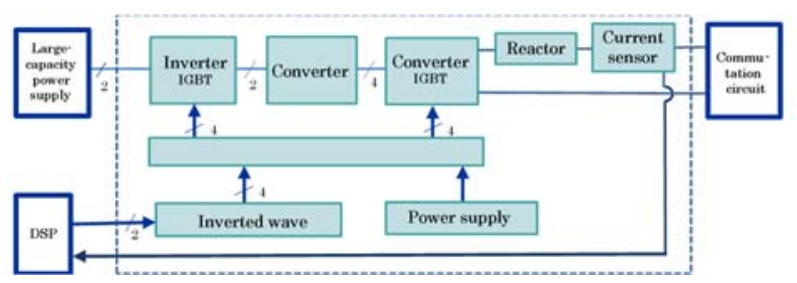

Fig. 12. Architecture of the constant current power supply.

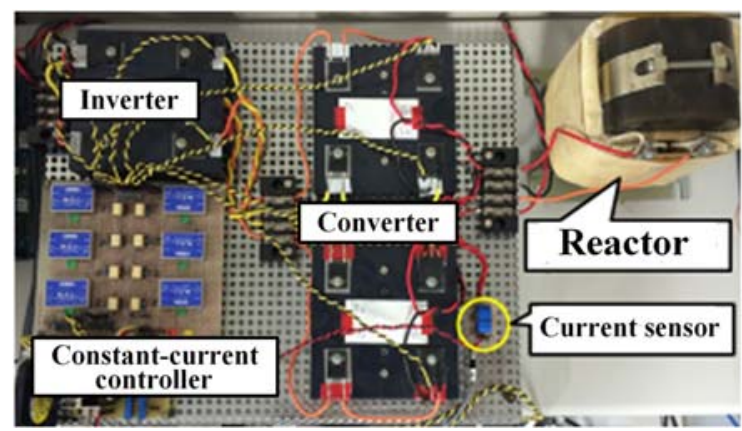

Fig. 13. A constant current power supply.
$\mathrm{S}_{2}, \mathrm{~S}_{3}$ which are switches for the converter are also operated in the same way.

\section{Construction of the Control Circuit}

\subsection{Driving Circuit for the Novel Motor}

Driving circuit for the motor consists of motor driver and Digital Signal Processor (DSP) as illustrated in

Fig. 10. Control signal of the current is generated in the DSP by using information from current sensors. Signal from rotary encoder is also utilized in order to calculate the control signal.

\subsection{Test Equipment of the Novel Motor}

Test Equipment used in this study is indicated in Fig. 11. DC motor which is used for the load and the novel motor are jointed via coupling. Rotary encoder is fixed on the shaft of the novel motor. The load test is carried out by connecting resistance to the DC motor. The DC motor is operated as a generator connecting with the variable load. The no load test is made by decoupling DC motor and the novel motor. The coupling of the rotary encoder and the motor is not removed, because the rotary encoder is necessary to detect the position of the rotor and the stator of the novel motor.

\subsection{Assembling of the Commutation Circuit}

Each circuit of the commutation circuit is in the inside of the dotted line in Fig. 10. IGBTs are employed for the switches. Control signal on the transmission lines is generated by the DSP analyzing the signal from the rotary encoder. The two signal lines to the switches are used in the case of the two-layer motor. Usually, four lines are required to control switches, but in this case, only two lines are used. Because one of A and B layer windings is always active, it is only necessary to assign the layer by the control signal. The control signal for each switch is generated in the inverted signal generator by using the DSP command.

\subsection{Construction of the Constant-Current Power Supply}

An architecture of the constant current power supply is illustrated in Fig. 12. Fig. 13 is a picture of the equipment. The switching semiconductor device is IGBT(GT50J325) which is also employed to the commutating circuit. Fast recovery diode(RURG5060) 


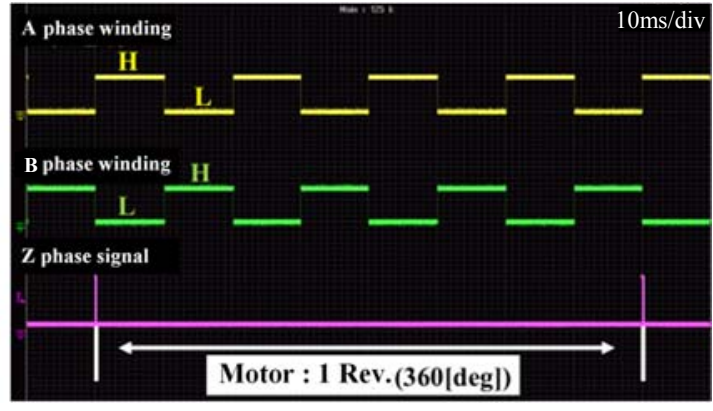

Fig. 14. Control signal of commutator for the first layer.

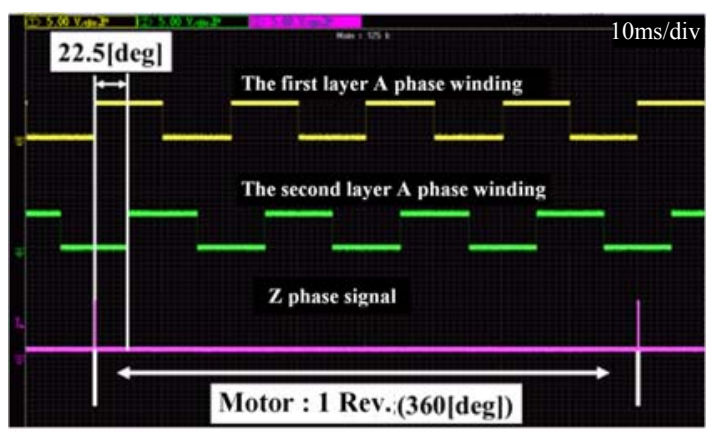

Fig. 15. Delay of the control signal of each layer.

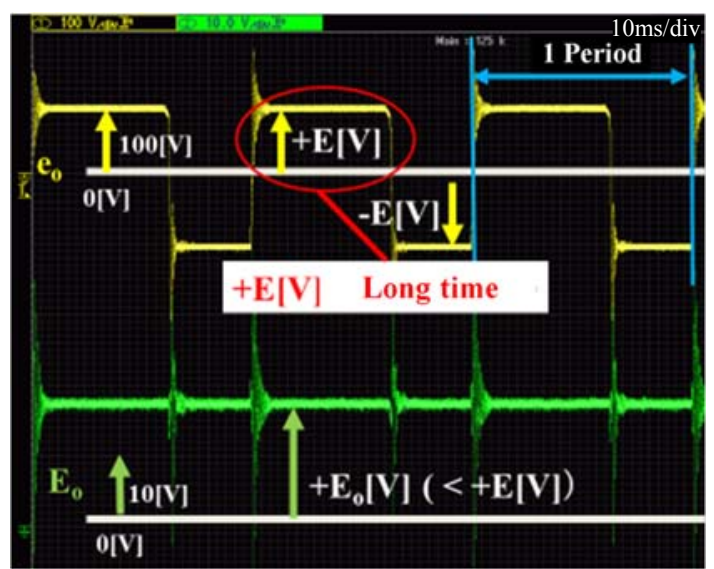

Fig. 16. Output of the constant current power supply.

which has fast response property is used for the diode of the converter. The output current is measured by current sensor after the reactor. The measured value is sent to the DSP in order to use the analysis for derivation of the

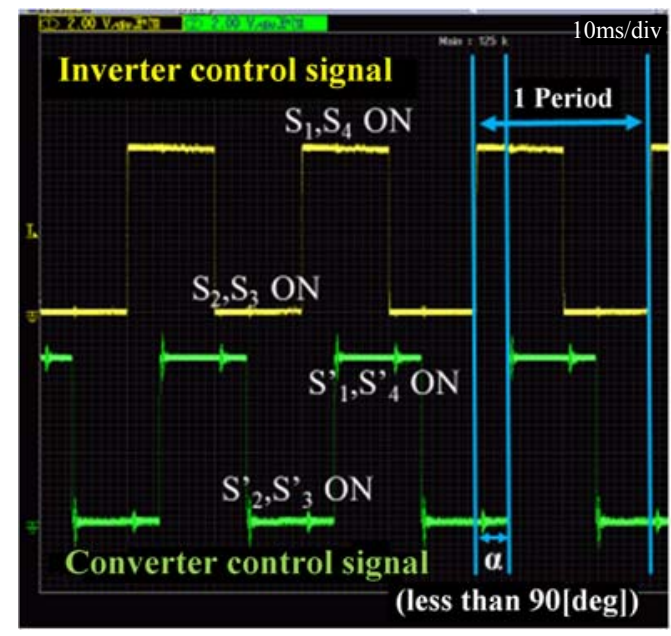

Fig. 17. Control signals of inverter and converter.

signal that is utilized to control the converter.

\section{Results of Experiments}

\subsection{Test of Driving Circuit}

In order to confirm the generation of semiconductor switching control signal, DC motor had been used instead of the novel motor. The DC motor was connected output shaft and rotated clockwise direction. Fig. 14 and Fig. 15 show results of the experiments. Fig. 14 shows the control signal of the first layer and Fig. 15 indicates the delay of the control signal of each layer, respectively. Concerning each winding of the novel motor's layer, A and B phase winding are not $\mathrm{ON}$ or OFF simultaneously. The upper part of Fig. 14 indicates the control signal of the A phase winding. The middle part shows the control signal of the B phase winding. If the signal is high level, IGBTs turn $\mathrm{ON}$ and current flows in the winding. While if the signal is low, IGBTs turn OFF and current doesn't flows. The lower part of Fig. 14 indicates the $\mathrm{Z}$ signal of the rotary encoder. A pulse is generated when the rotary encoder, i.e. the motor, makes a round. Fig. 15 indicates that the $\mathrm{Z}$ signal rises at the point of $\theta$ equals to zero to $0.35[\mathrm{deg}]$. The upper part of Fig. 15 shows control signal of A phase winding in the first layer, the middle part indicates A phase winding in the second layer and the lower part designates the $\mathrm{Z}$ signal of the rotary encoder. If the novel motor is driven with no problem, the control signal of the commutator becomes square wave as shown in Fig. 7. While Fig. 14 shows that A phase windings turn ON and 
OFF alternatively, never turn ON simultaneously, by the correct control signals. On the other hand, Fig. 15 indicates that control signal of the second layer is delayed $22.5[\mathrm{deg}]$ from the first layer as shown in Fig. 7. Therefore, the control signal of each layer is generated correctly.

\subsection{Driving Test of Constant Current Power Supply}

Driving test had been made by connecting resistance (3.3 [ $\Omega] 100[\mathrm{~W}])$ to the output terminal of assembled constant-current power supply. Output voltage and the control signals, in the case of output is 5[A], are shown in Fig. 16 and Fig. 17. The upper part of Fig. 16 shows $e_{0}$ and the lower part is $E_{0}$. Fig. 17 indicates the control signals of inverter and converter. In Fig.16, the time which $e_{0}$ equal to the source voltage $+E$ is longer than the time which $e_{0}$ equal to $-E$ in a period. As a result, output which is smoothed by reactor became positive. In Fig. 17, As $\alpha$ of the control signal for inverter and converter is smaller than the angle that makes $E_{0}$ zero, the output become positive value. In conclusion, desirable operation of the constant-current power supply had been confirmed.

\section{Conclusions}

In this research, we developed the drive circuit for novel motor which is one of an improved SRM. For the novel motor, some driving methods are considered, such as chopper method, phase control method and inverter method. The chopper method that is realized by simple hardware is often employed for the constant current power supply. Because of the complicated hardware, the phase control method is rarely utilized. While the method makes it possible to control the current precisely. In addition, its circuit can be effectively controlled by using microprocessor. Then, this paper presents phase control method. For that purpose, we have prototyped equipment which includes constant current power supply and commutator circuit. Those equipment is tested individually to confirm desirable operations. After that, driving test of the novel motor had been carried out by using various equipment. The driving circuit have been operated without fatal error. But the results of the experiments show that there are room for improvement in some point of view. As each phase winding is connected in serial, for example, each current in the winding can't be controlled independently. The effective regenerative operation hasn't worked yet. We will modify the circuit for more efficient operation and develop the motor system in order to produce the EV without rare earth materials.

\section{References}

(1) S. Tsugawa, "Special issue on fundamental technologies for ITS,” JRM Vol.13, No. 4, pp. 339, 2001

(2) R. Hagura and S. Kitayama, "Development of energy management of hybrid electric vehicle for improving fuel consumption via sequential approximate optimization,” JRM Vol.26, No. 5, pp. 600-606, 2014

(3) Y. Takahashi, M. Tomike and T. Hirayama, "pico-FCV: small single-operator electric-vehicle using $20 \mathrm{~W}$ hydrogen fuel cell developed for mechatronics education,” JRM Vol. 26, No. 4, pp.515-517, 2014

(4) H. Sakamoto, "Design and manufacture of small-sized electric vehicles for mechanical engineering education," JRM Vol. 13, No. 4, pp.426-431, 2001

(5) Y. Takeda, S. Morimoto, N. Matsui, Y. Honda, "Design and control of interior permanent synchronous motor," Ohmsha, pp. 2-3, 2001

(6) M. N. Uddin and M. A. Rahman, "Fuzzy logic based speed control of an IPM synchronous motor drive," JACIII Vol. 4, No. 3, pp.212-219, 2000

(7) K. Abe, H. Haga, K. Ohishi and T. Hiraide, "Harmonics reduction control for the input current of electrolytic capacitor-less high-power-factor inverter for IPMSM," IEEJ Jounal of Industry Applications, Vol. 4, No. 3, pp. 116-125, 2015

(8) S. Smaka, "Fast analytical model for switched reluctance machines," IEEJ Jounal of Industry Applications, Vol. 4, No. 4, pp. 352-359, 2015

(9) Y. Ishihara, M. Sugiura, H. Ishikawa and H. Naitoh, "Improving the efficiency of switched reluctance motors using a step-skewed rotor," IEEJ Jounal of Industry Applications, Vol. 4, No. 4, pp. 445-453, 2015 